\section{Lawsonia intracellularis in Pigs: Progression of Lesions and Involvement of Apoptosis}

\author{
Roberto M.C. Guedes', Mariana A. Machuca², Maria A. Quiroga ${ }^{2}$, \\ Carlos E. Real Pereira', Talita P. Resende ${ }^{3}$, and Connie J. Gebhart ${ }^{3}$
}

Veterinary Pathology

2017, Vol. 54(4) 620-628

(C) The Author(s) 2017

Reprints and permission:

sagepub.com/journalsPermissions.nav DOI: 10.1 177/03009858|7698206

journals.sagepub.com/home/vet

(9)

\begin{abstract}
The purpose of this study was to follow the progression of gross and histologic lesions and apoptosis events in Lawsonia intracellularis-infected enterocytes through the course of the disease, proliferative enteropathy (PE). Thirty 5-week-old pigs were divided into 2 groups: 20 challenged and 10 control animals. Groups of 3 pigs, 2 challenged and I control, were euthanized at I, 3 , $5,8,11,15,19,24,29$, and 35 days after inoculation. Complete necropsies were performed with gross evaluation. Tissue samples from different sites of the gastrointestinal tract and other visceral organs were collected for routine histologic staining and for immunohistochemistry (IHC) for L. intracellularis. In addition, caspase-3, terminal deoxyuridine nick-end labeling assay, and electron microscopy were performed in ileum samples. Macroscopic and histologic lesions suggestive of PE were first detected II days after infection and continued through day 24. L. intracellularis antigen was first detected in the intestine by IHC on day 5 after inoculation, and the bacterium was first detected by transmission electron microscopy on day I5. Positive IHC staining for [L. intracellularis] and enterocyte proliferation, but no gross lesion, were detected on day 29. All 3 pigs euthanized on day 35 were grossly and histologically normal and IHC negative. Hyperplastic crypts in challenge pigs had more apoptotic cells on days I5, 19 , and 24 postinfection $(P<.05)$ compared to control pigs. Our results demonstrated the progression of lesions and infection by $L$. intracellularis and that inhibition of enterocyte apoptosis is not involved in the pathogenesis of proliferative enteropathy.
\end{abstract}

\title{
Keywords
}

apoptosis, caspase-3, immunohistochemistry, Lawsonia intracellularis, pathogenesis, proliferative enteropathy, swine, TUNEL

Porcine proliferative enteropathy $(\mathrm{PE})$ is an intestinal infectious disease characterized by thickening of the aboral small intestinal mucosa due to enterocyte proliferation associated with the presence of an intracellular bacterium, Lawsonia intracellularis. ${ }^{16,19}$ Proliferative enteropathy has been reported in several different animal species ${ }^{3,25}$ but has been best described in pigs and hamsters. The disease has been recognized in all major swine industries throughout the world.

Cell proliferation, an important feature of PE, cannot be reproduced in vitro ${ }^{15,20}$; therefore, in vivo studies provide a better understanding of its pathogenesis. An increased antiapoptotic gene expression and a decreased pro-apoptotic gene expression have been found at 21 days postinfection, ${ }^{24}$ and temporary reduction of apoptosis induced by $L$. intracellularis infection is hypothesized to be one of the mechanisms involved in enterocyte proliferation. At late stages of the infection, the disappearance of the bacteria in enterocytes was associated with resumption of apoptotic events and the histologic appearance of the intestinal mucosa returns to normal. ${ }^{21}$ However, no quantitative or statistical evaluations of the apoptotic events were performed in affected and nonaffected PE cases. Although a PE progression study has been performed focusing on immune response, ${ }^{17}$ apoptotic events were not investigated in vivo. A more systematic study of the relationship between enterocyte proliferation and apoptotic events would enhance the knowledge of the mechanism of cell proliferation observed in PE lesions.

The objective of this study was to investigate the progression of gross and histologic lesions and apoptotic events in association with the presence of $L$. intracellularis bacteria through the course of disease in experimentally infected pigs.

\section{Materials and Methods}

Preparation of the $\mathrm{L}$. intracellularis Inoculum

Pure culture of the pathogenic L. intracellularis isolate PHE/MN1-00 was used as the inoculum material. The

\footnotetext{
'Veterinary School, Department of Clinic and Surgery, Universidade Federal de Minas Gerais, Belo Horizonte, Minas Gerais, Brazil

${ }^{2}$ Department of Veterinary Pathology, Faculty of Veterinary Science, La Plata National University, Buenos Aires, Argentina

${ }^{3}$ Department of Veterinary and Biomedical Sciences, College of Veterinary Medicine, University of Minnesota, Saint Paul, MN, USA
}

\section{Corresponding Author:}

Roberto M. C. Guedes, Veterinary School, Department of Clinic and Surgery, Universidade Federal de Minas Gerais, PO Box 567, Belo Horizonte, Minas Gerais 31.270-90I, Brazil.

Email: guedes@vet.ufmg.br 
bacterial growth conditions were the same as described elsewhere. ${ }^{8,9}$ Briefly, eukaryotic cells were infected with L. intracellularis, grown for 7 days in a microaerophilic environment and then passed to expand the culture. At the last passage, bacteria were released from infected eukaryotic cells, concentrated by centrifugation, suspended in sucrosepotassium glutamate (SPG) solution, and kept at $4^{\circ} \mathrm{C}$ until challenging pigs later on the same day.

Quantification of the pure culture inoculum was accomplished by making serial 1:10 dilutions of the inoculum, coating 15-well glass slides, and staining with the monoclonal antibody specific for L. intracellularis as described elsewhere. ${ }^{10}$ Aliquots of the pure culture inoculum were also submitted to the University of Minnesota Veterinary Diagnostic Laboratory for bacteriologic and virologic analyses.

\section{Study Design}

Thirty 5-week-old pigs of mixed sex weighing between 9 and $13.6 \mathrm{~kg}$ were obtained from a commercial herd with no history or recorded cases of PE. The herd was serologically negative for L. intracellularis, porcine respiratory and reproductive syndrome, and Actinobacillus pleuropneumoniae. Segregated early weaning at 16 days of age to an off-site nursery was routinely performed on the farm to help minimize respiratory disease.

Two days before challenge, serum and fecal samples were collected from all pigs and tested by the immunoperoxidase monolayer assay (IPMA) ${ }^{11}$ and PCR for IgG and L. intracellularis DNA, ${ }^{13}$ respectively, to assure PE-negative status. One day before challenge, the animals were divided into 2 groups randomized by weight: 10 pigs in the control group and 20 pigs in the challenged group. The pigs were allocated into 3 different rooms in isolation barns at the University of Minnesota under restrictive biosecurity protocol, and the control group was always managed first.

On day 0 , pigs in the control group received SPG with 5\% FBS solution, and each pig in the challenged group received $4.37 \times 10^{9}$ organisms of the L. intracellularis isolate PHE/ MN1-00 in SPG with 5\% FBS solution. All animals were intragastrically dosed with $31 \mathrm{ml}$ of the respective inoculum using a stomach tube. Health observations based on the consistency of the feces graded from 1 (normal) to 5 (bloody and liquid), as described elsewhere, ${ }^{9}$ were made 3 times per week. Groups of 3 randomly chosen animals, 2 challenged and 1 nonchallenged, were euthanized at different times after the inoculation as follows: $1,3,5,8,11,15,19,24,29$, and 35 days. All procedures were conducted in accordance with the guidelines of the Animal Care and Use Manual of the University of Minnesota and were approved by the Institutional Animal Care and Use Committee (A3456-01).

\section{Necropsy and Sampling}

Complete necropsies were performed immediately postmortem. Fecal samples were collected from the rectum of all pigs at each necropsy date and tested for L. intracellularis shedding by PCR ${ }^{13}$ Intestinal lavage of the aboral $25 \mathrm{~cm}$ of the small intestine (ileum) of all pigs euthanized after day 11 was made with $20 \mathrm{ml}$ of cold PBS. The lavage was then centrifuged at 150 $\times g$ for 5 minutes to eliminate solid material, and the supernatant was frozen for secretory IgA titration using a modified IPMA test. ${ }^{7}$ Severity and lesion length were evaluated and graded from 0 (normal) to 4 (hemorrhagic), as described elsewhere. ${ }^{12}$ Tissue samples from tonsil, stomach, duodenum, middle jejunum, ileum, cecum, proximal colon, spiral colon, rectum, ileocecal and hepatic lymph nodes, liver, spleen, kidney, lung, and heart were collected, fixed in $10 \%$ neutral buffered formalin, and histologically processed. Ileum samples that were adjacent to sections selected for light microscopy were trimmed in small fragments immediately after euthanasia and placed in Karnovsky's solution (2.5\% glutaraldehyde, $1 \%$ paraformaldehyde in PBS, $\mathrm{pH}$ 7.2). After 30 minutes, when tissue had hardened, small $(1-\mathrm{mm})$ pieces were cut, fixed for 90 more minutes, washed 3 times with PBS (pH 7.2), and kept at $4^{\circ} \mathrm{C}$ until further processing for transmission electron microscopy examination.

\section{IgA Titration in Intestinal Lavage}

IgA titration was performed as previously described. ${ }^{7}$ Briefly, 96-well plates (Nunc, Rochester, NY) containing acetonefixed monolayers of McCoy cells heavily infected with $L$. intracellularis were rehydrated in a solution of PBS with 5\% skimmed milk for 10 minutes at $37^{\circ} \mathrm{C}$ to block nonspecific reactions. The intestinal lavage samples were diluted in the same block solution in serial 4-fold dilutions (1:4, 1:16, $1: 64$, and 1:256). Then, $50 \mathrm{ml}$ of each diluted sample was added to the test well. The plate was incubated for 30 minutes at $37^{\circ} \mathrm{C}$ and then washed 5 times with PBS containing $0.05 \%$ Tween 20 (PBST, Tween 20 obtained from Sigma, St Louis, MO). Goat antiporcine IgA-peroxidase conjugate (Bethyl Laboratories, Montgomery, TX), diluted 1:1000 in PBST, was added at a concentration of $30 \mathrm{ml} /$ well, and incubation proceeded for 45 minutes at $37^{\circ} \mathrm{C}$. The plate was washed 5 times with PBST and $100 \mathrm{ml}$ prediluted chromogen (3-amino-9-ethyl-carbazole; Sigma) solution was added to each well and incubated at room temperature for 20 minutes. The plate was washed with PBS 3 times, allowed to dry, and examined using an inverted light microscope.

\section{Histology, Immunohistochemistry, and TUNEL Assay}

All formalin-fixed tissue samples were stained by HE and by IHC stain (peroxidase-labeled streptavidin method; Dako, Carpinteria, CA) using a monoclonal antibody specific for L. intracellularis. ${ }^{16}$ Lesions of PE were scored from 0 to 3 according to histopathologic changes, considering score 0 when no crypt with hyperplastic enterocytes was present and score 3 when all crypts were hyperplastic. Table 1 shows the microscopic features of the graded score system applied. 
Table I. Incidence of Mucosal Hyperplasia and Bacterial Antigen Labeling in Different Intestinal Segments in Pigs Experimentally Inoculated (2 pigs at each date) With Pure Culture of Lawsonia intracellularis. ${ }^{\text {a }}$

\begin{tabular}{lcccccccccc}
\hline & \multicolumn{1}{c}{ No. of Days After Inoculation } \\
\cline { 2 - 10 } Tissue Section & $\mathrm{I}$ & 3 & 5 & 8 & II & 15 & 19 & 24 & 29 & 35 \\
\hline Duodenum & $0 / 0$ & $0 / 0$ & $0 / 0$ & $0 / 0$ & $0 / 0$ & $0 / 1$ & $0 / 0$ & $0 / 0$ & $0 / 0$ & $0 / 0$ \\
Jejunum & $0 / 0$ & $0 / 0$ & $0 / 2$ & $0 / 1$ & $2 / 1$ & $1 / 1$ & $1 / 1$ & $1 / /$ & $0 / 0$ & $0 / 0$ \\
Ileum & $0 / 0$ & $0 / 0$ & $0 / 1$ & $0 / 2$ & $2 / 2$ & $1 / 2$ & $2 / 2$ & $1 / 1$ & $0 / 1$ & $0 / 0$ \\
Cecum, proximal & $0 / 0$ & $0 / 0$ & $0 / 0$ & $0 / 2$ & $2 / 2$ & $1 / 2$ & $2 / 2$ & $2 / 2$ & $1 / 2$ & $0 / 0$ \\
$\quad$ colon & & & & & & & & & & \\
$\quad$ Spiral colon & $0 / 0$ & $0 / 0$ & $0 / 0$ & $0 / 0$ & $0 / 1$ & $1 / 2$ & $1 / 2$ & $1 / 1$ & $0 / 1$ & $0 / 0$ \\
Rectum & $0 / 0$ & $0 / 0$ & $0 / 0$ & $0 / 0$ & $0 / 1$ & $0 / 1$ & $2 / 2$ & $1 / 1$ & $1 / 1$ & $0 / 0$ \\
\end{tabular}

${ }^{a}$ Data are given as number of pigs with hyperplastic enterocytes/number of pigs with $L$. intracellularis antigen labeling.

Only ileum sections were stained by IHC using a rabbit polyclonal anti-active caspase-3 antibody (Research Diagnostics, Inc., Flanders, NJ) at 1:100 dilution and using a steamer for 20 minutes for antigen retrieval for quantification of apoptotic cells. Cells undergoing apoptosis were also identified by using the terminal deoxyuridine nick-end labeling (TUNEL) method, with a commercial apoptosis detection kit (ApopTag ${ }^{\mathbb{R}}$ Plus Peroxidase In Situ Apoptosis Detection Kit; Serologicals Corporation, Atlanta, GA). In brief, the procedure was as follows: multiple fragmented DNA $3^{\prime}-\mathrm{OH}$ ends on the sections were labeled with digoxigenin-dUTP in the presence of terminal deoxynucleotidyl transferase (TdT). Peroxidase-conjugated antidigoxigenin antibody was reacted with the sections and apoptotic nuclei were visualized by peroxidase-diaminobenzidine (DAB) reaction. The sections were then counterstained with methylgreen. Negative control slides were treated identically, but the enzyme solution was omitted. Apoptotic indices (AIs) for caspase-3 and TUNEL assays were calculated using the number of crypt apoptotic cells positively stained / total cells counted $\times 100$. Four randomly chosen fields per section ( 5 crypts/field) were observed.

\section{Transmission Electron Microscopy}

Ileum samples collected for electron microscopy evaluation, previously washed in PBS and kept refrigerated, were fixed in $1 \%$ osmium tetroxide, dehydrated through a graded series of alcohol, and embedded in resin. Ultrathin sections were cut with a diamond knife and supported on 200-nm copper grids. After staining with uranyl acetate and lead citrate, the sections were examined in a Phillips CM12 transmission electron microscope.

\section{Statistical Analysis}

Mann-Whitney nonparametric test was used to compare AIs between challenge and control groups, with significance at $P<.05$. Pearson correlation analysis between TUNEL and caspase-3 IHC was performed.

\section{Results}

\section{Clinical Findings}

Clinical signs characterized by soft feces and watery diarrhea occurred only in challenged pigs and began 9 days after inoculation. Blood-tinged watery feces were observed in 2 to 3 consecutive clinical evaluation days in 3 challenged pigs from days 13 to 28 after inoculation. One of these animals became progressively depressed and gaunt for 10 days and it had to be euthanized on day 24 after challenge. This was the only animal not randomly chosen for euthanasia.

\section{Macroscopic Lesions}

Gross lesions were restricted to the alimentary tract, abdominal cavity, and mesenteric lymph nodes. Mildly increased thickness and hyperemia of the mucosa (grade 1 lesion) extending primarily from the ileocecal orifice for $5 \mathrm{~cm}$ was seen on day 11 in 1 challenged pig (Fig. 1).

At necropsy on day 15,1 challenged animal with blood-tinged watery diarrhea had enlarged lymph nodes 3 times the normal size and approximately $200 \mathrm{ml}$ of translucent yellowish transudate in the abdominal cavity. The serosa of the ileum extending up to the middle jejunum was corrugated and the intestinal wall was thicker than normal. The ileal mucosa extending up to 1 meter in the distal jejunum and $5 \mathrm{~cm}$ into the colon was corrugated with thick, evident, and hyperemic folds (grade 2 lesions) and fibrin covering the surface (Fig. 2). Lesions extending for 2 meters in the middle jejunum were more severe than in the ileum and distal jejunum with small superficial areas of necrosis of the mucosa and formation of fibrinonecrotic membranes (grade 3 lesion). The spiral colon contained dark brown, blood-tinged, watery feces.

By day 19, 1 pig had grade 2 lesions in the ileum extending 1.3 meters to the distal jejunum and $60 \mathrm{~cm}$ more of grade 1 lesions in the adjacent proximal middle jejunum. The pig that was euthanized on day 24 was pale and dehydrated and had prominent spine, rib, and pelvic bones. It had grade 2 lesions in the ileum extending 2.2 meters in the distal jejunum and $10 \mathrm{~cm}$ in the cecum and colon. Intestinal lesions (1 meter long) were more severe (grade 3 ) with extensive necrosis of the mucosa in the adjacent middle jejunum (Fig. 3). The spiral colon contained dark brown, blood-tinged, watery feces.

No gross lesions were observed in either of the challenged animals necropsied on day 29. On day 35, ileum from 1 of the challenged pigs appeared thicker than normal with slightly corrugated mucosa. Control pigs had no macroscopic lesions at any of the necropsy time points.

\section{Histopathology and IHC for L. intracellularis}

Histologic lesions associated with PE occurred in the intestinal tract and ileocecal lymph nodes. Several challenged and control pigs had mild interstitial pneumonia and mild to moderate hepatic degeneration. No L. intracellularis antigen was detected in the heart, kidney, liver, spleen, lung, and stomach sections of any challenged or control animal by IHC. 

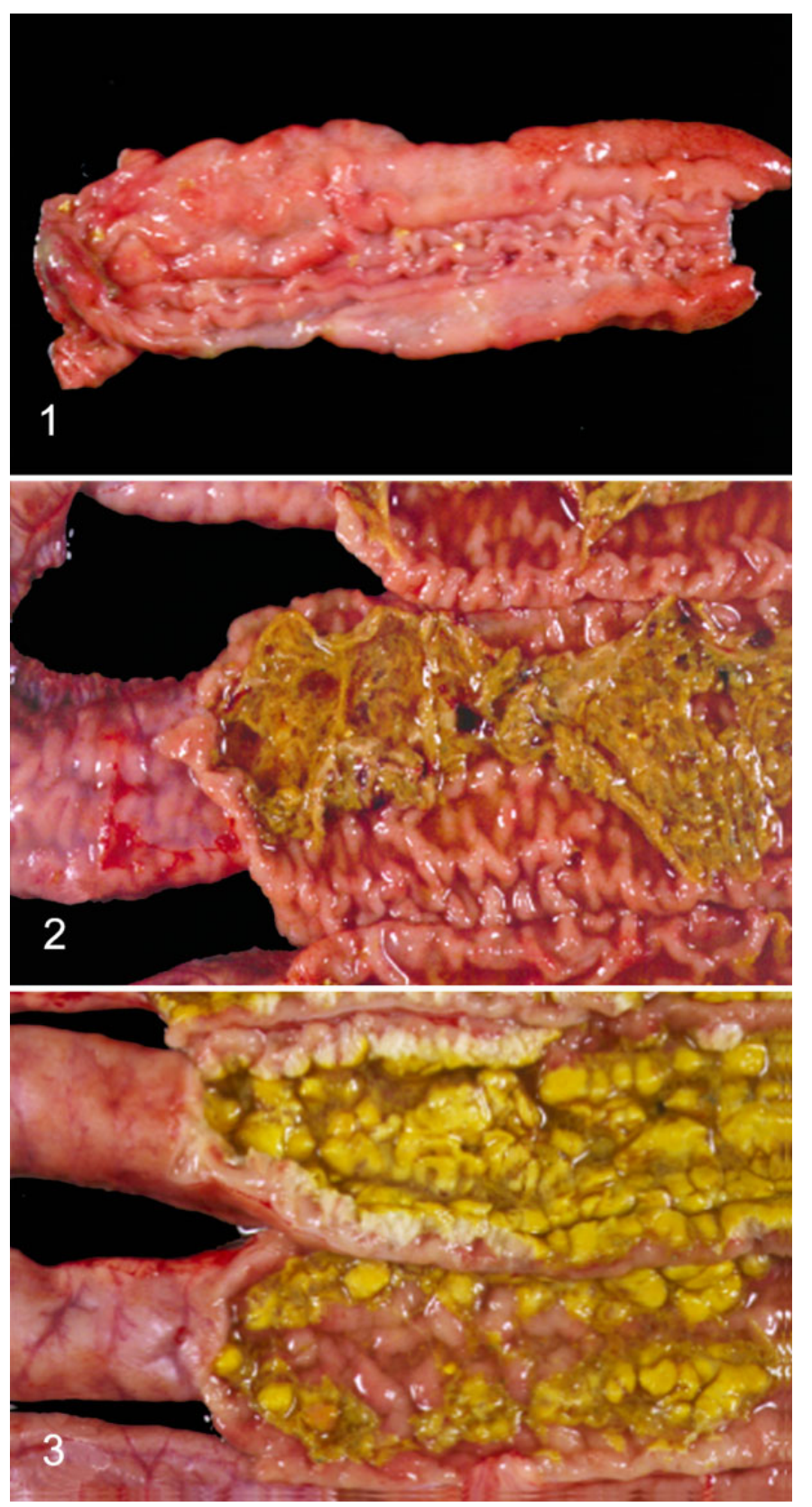

Figures I-3. Proliferative enteropathy, ileum, pig. Figure I. Corrugation of the mucosa and hyperemia of folds, day II. Figure 2. Corrugation of the serosa and mucosa and deposition of fibrin forming a pseudomembrane, day 15. Figure 3. Thickening of the intestinal wall and extensive necrosis of superficial mucosa, day 24 .

L. intracellularis antigen was first detected by IHC in the intestine 5 days postinoculation when both challenged pigs had isolated foci of bacteria in the cytoplasm of crypt enterocytes in the jejunum (Table 1) and 1 of these pigs also had L. intracellularis antigen in 1 crypt in the ileum. No histologic lesion was noted in association with presence of the bacterial antigen. By day 8, similar focal areas of $L$. intracellularis-labeled antigen were observed in crypt enterocytes in the ileum and proximal colon of 1 pig with no concurrent histologic alteration. The second challenged pig euthanized on day 8 had multifocal positive antigen-labeled bacteria in crypt enterocytes and in the tip of villi in the ileum (Fig. 4b) and jejunum. Isolated foci of bacteria were also detected in the cecum and proximal colon. Histologically, there was discrete villous atrophy in the ileum section, but no sign of enterocyte proliferation was observed (Fig. 4a).

Multifocal crypt cell hyperplasia and a reduced number of goblet cells were evident in ileum and proximal colon sections in both challenged pigs on day 11 . Crowded crypt-type epithelium covered the lower one-half to three quarters of each villus. Villi were also wider than normal because hyperplastic epithelial cells were elongated and pseudostratified. Some crypts were mildly dilated and crypt lumens contained cell debris. No evident inflammation was found. Focal areas of crypt hyperplasia were also observed in the jejunum of both animals. Intense L. intracellularis antigen label was observed in the cytoplasm of hyperplastic crypts and also in the cytoplasm of mononuclear cells in the lamina propria in the ileum. Multifocal areas in the jejunum and focal areas in the proximal and spiral colon, cecum, and rectum had bacterial antigen labeled in the cytoplasm of epithelial cells.

By day 15, immature epithelial cell hyperplasia was diffuse in the ileum (Fig. 5a), cecum, proximal and spiral colon, and jejunum. Affected areas of the jejunum and ileum were associated with severe villous atrophy. Necrosis of the superficial mucosal overlayer and deposition of fibrin forming a fibrinonecrotic membrane were observed in the jejunum, whereas intense and diffuse bacterial antigen labeling was found in crypt cells and lamina propria in the ileum (Fig. 5b) and jejunum, and lumen and cytoplasm of crypt cells in the duodenum, proximal and spiral colon, cecum, and rectum. Bacterial antigen was also detected in the lymphoid nodules of submucosa of the proximal colon. Similar histologic lesions and L. intracellularis distribution were observed on days 19 and 24 (Fig. 6a, b), except that more severe and deeper mucosal necrosis was detected in jejunum sections on day 24 .

By day 29, epithelial cell hyperplasia was observed only in the rectum and proximal colon of the 2 necropsied pigs (Fig. 7a). Multifocal areas of L. intracellularis-labeled antigen were visualized in the cytoplasm of crypt cells and in the lamina propria in the rectum (Fig. 7b) and only in focal areas in enterocytes or in the lamina propria in the ileum, cecum, and proximal and spiral colons. It appeared that most of the bacterial infection had been cleared from enterocytes and bacterial antigen was more often present in the cytoplasm of mononuclear cells in the lamina propria. By day 35, no histologic lesions or L. intracellularis-labeled antigens were observed in the intestinal sections.

Ileocecal lymph nodes had prominent germinal centers and medullary cords filled with plasma cells from days 5 to 24 after inoculation. Cortical and medullary sinuses usually contained some neutrophils. Multiple foci of $L$. intracellularis-labeled antigen were detected in the cortical and medullary sinuses from days 8 to 24 after inoculation. Small foci of bacterial antigen label were observed in the cytoplasm of mononuclear 

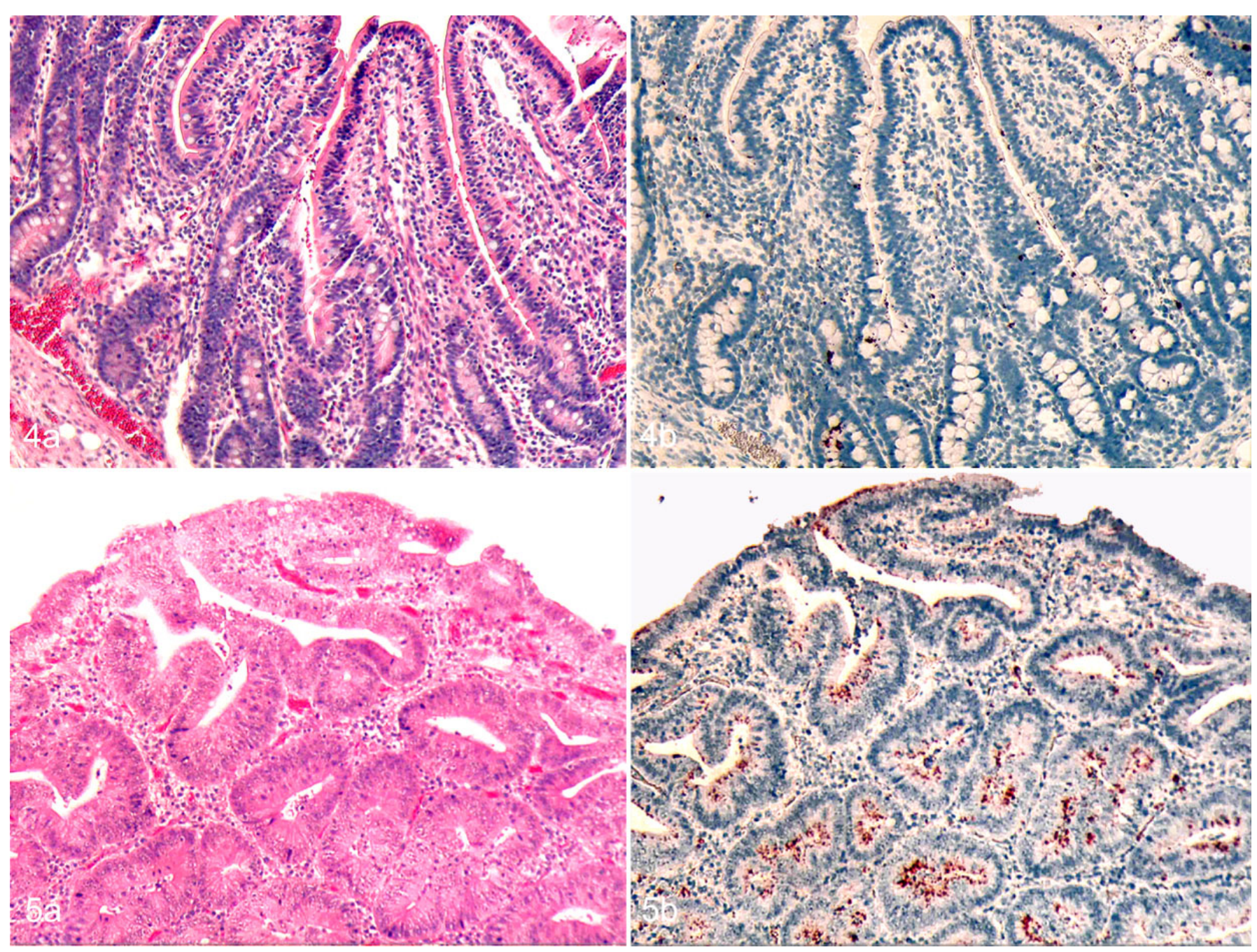

Figures 4, 5. Proliferative enteropathy, intestine, pig. Figure 4. Day 8, (a) normal ileum. Hematoxylin and eosin (HE). (b) Low amount of Lawsonia intracellularis antigen in the cytoplasm of enterocytes. Immunohistochemistry (IHC) for L. intracellularis. Figure 5. Day I5, (a) ileum with hyperplastic crypts. HE. (b) Bacterial antigen in the cytoplasm of enterocytes and in a few mononuclear cells in the lamina propria. IHC for $L$. intracellularis.

cells in the same regions as ileocecal lymph nodes in the hepatic lymph node on days 8 and 19 after inoculation.

Clusters of $L$. intracellularis antigen were observed in the lumen of isolated tonsil crypts and in the cytoplasm of crypt epithelial cells and mononuclear cells adjacent to the crypt on days 19 and 35 after inoculation.

\section{TUNEL and Caspase-3}

The apoptotic index (AI) is shown in Fig. 8, both for TUNEL and caspase- 3 IHC in control and challenged pig groups. In the control samples, the AI remained constant along the evaluated period. In samples from challenged pigs, the AI increased from days 11 to 24 after challenge, with $P<.05$ at $11,15,19$, and 24 dpi (Figs. 9, 10), and returned to the control values at day 29 after challenge. In parallel, the lesion scores were highest at 15 days postchallenge. A high correlation $(r=.90)$ between the AIs was obtained using activated caspase- 3 and TUNEL.

\section{Transmission Electron Microscopy}

At 15,19 , and 24 days after inoculation, L. intracellularis organisms were evident within the cytoplasm of affected enterocytes in crypts and villi. Bacteria were straight or slightly curved cylindrical rods with crenulated cell walls measuring 0.1 to 0.3 micrometer in diameter. The organisms were most abundant just beneath the terminal web. Enterocytes parasitized by the bacteria had poorly developed microvilli (small, sparse, and irregularly distributed) (Figs. 11, 12). Some infected crypt cells were shrunken and protruding into the lumen.

\section{Fecal Shedding and Intestinal Lavage IgA Titers}

All fecal and serum samples collected before inoculation were negative. Fecal shedding and intestinal lavage IgA titer results are summarized in Table 2. Fecal shedding was detected as early as 3 days and up to 35 days after inoculation. IgA titers 


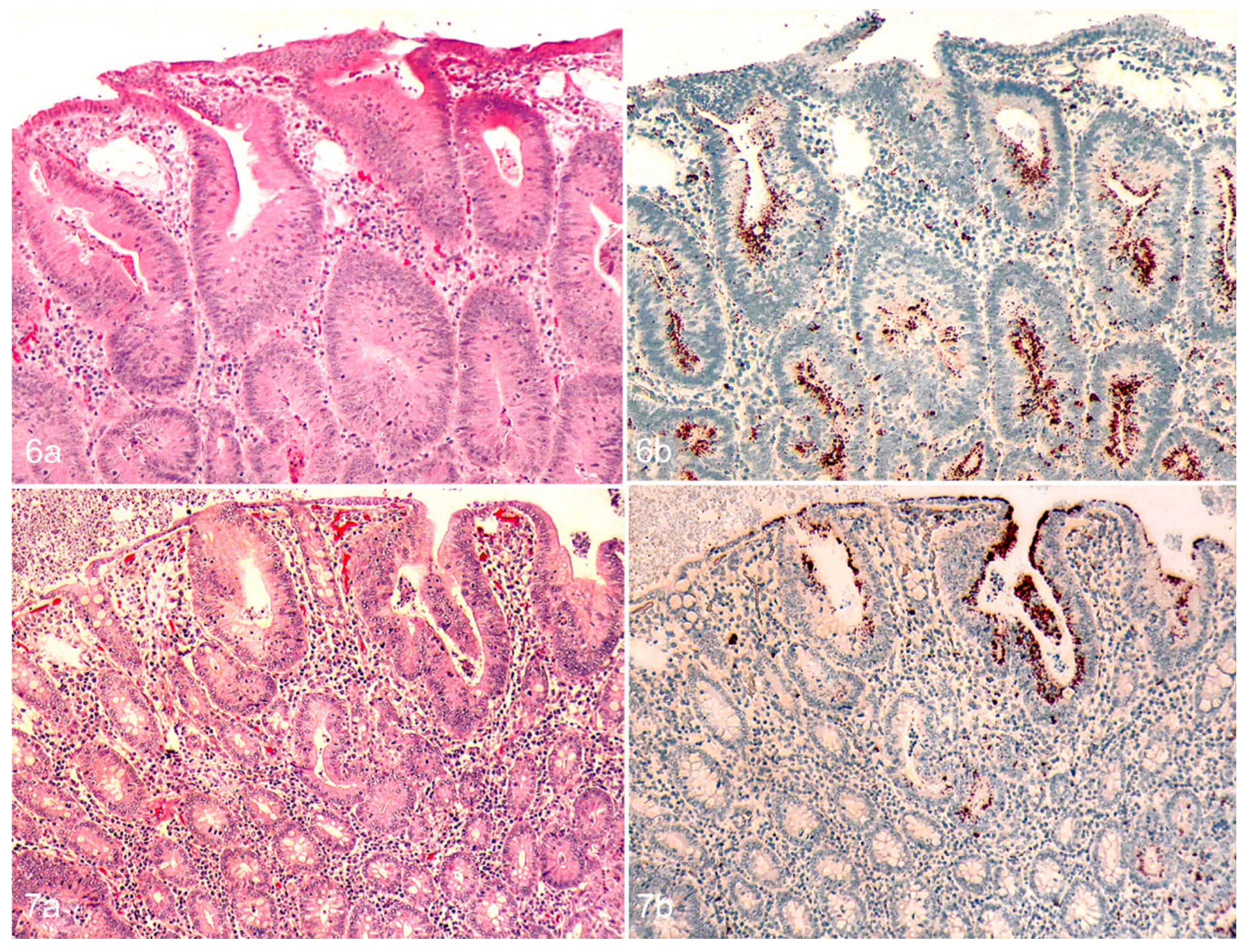

Figures 6, 7. Proliferative enteropathy, intestine, pig. Figure 6. Day 24, (a) ileum with hyperplastic crypts. HE. (b) Extensive amount of $L$. intracellularis antigen in the cytoplasm of enterocytes and in the lamina propria. IHC for L. intracellularis. Figure 7. Day 29, (a) rectum with isolated hyperplastic crypts. HE. (b) Bacterial antigen is present in the crypt epithelium. IHC for L. intracellularis.

in intestinal lavages were first detected at day 15 and were still detectable at day 29 , ranging from 1:4 to 1:16.

\section{Discussion}

In this study, the in vivo progression of gross and histologic lesions in pigs after infection with cell-cultured L. intracellularis up to 35 days after oral inoculation was followed. In this study, L. intracellularis antigen was first detected in crypt enterocytes in ileum and jejunum sections at day 5 , whereas MacIntyre et $\mathrm{al}^{17}$ and Boutrup et $\mathrm{al},{ }^{2}$ also using experimentally infected pigs, detected a very small amount of antigen as early as 3 days after inoculation. Nevertheless, in all 3 of these studies, the amount of L. intracellularis detected was similar around 5 days after infection.

Histologic lesions typical of PE were detected only on day 11 postinoculation. Mild circulatory changes suggestive of early infection with $L$. intracellularis were observed macroscopically by day 11 , but typical mucosal thickening and corrugation was seen only on day 15 postinoculation. Diarrhea was observed as early as 9 days and lasted until 28 days postinoculation, coinciding with the detection of the first macroscopic and microscopic PE lesions at 11 days postinoculation, consistent positivity in PCR fecal samples, and L. intracellularis antigens in the large intestine until 29 days postinoculation. The bacteria were detected by electron microscopy as well on this necropsy day. Macroscopic lesions were more often present in the small intestine, more specifically in the ileum and jejunum, but lesions in the proximal colon and cecum could occasionally be detected. Only histologic lesions of PE were present in the spiral colon and rectum. In another study in which the disease progression was also followed, ${ }^{17}$ the macroscopic lesions were milder and sporadic, being observed in only 4 of 16 experimentally inoculated pigs at days 13,14 , 35 , and 42 after challenge. Our results indicate that gross lesions related to PE were mainly found between days 15 and 


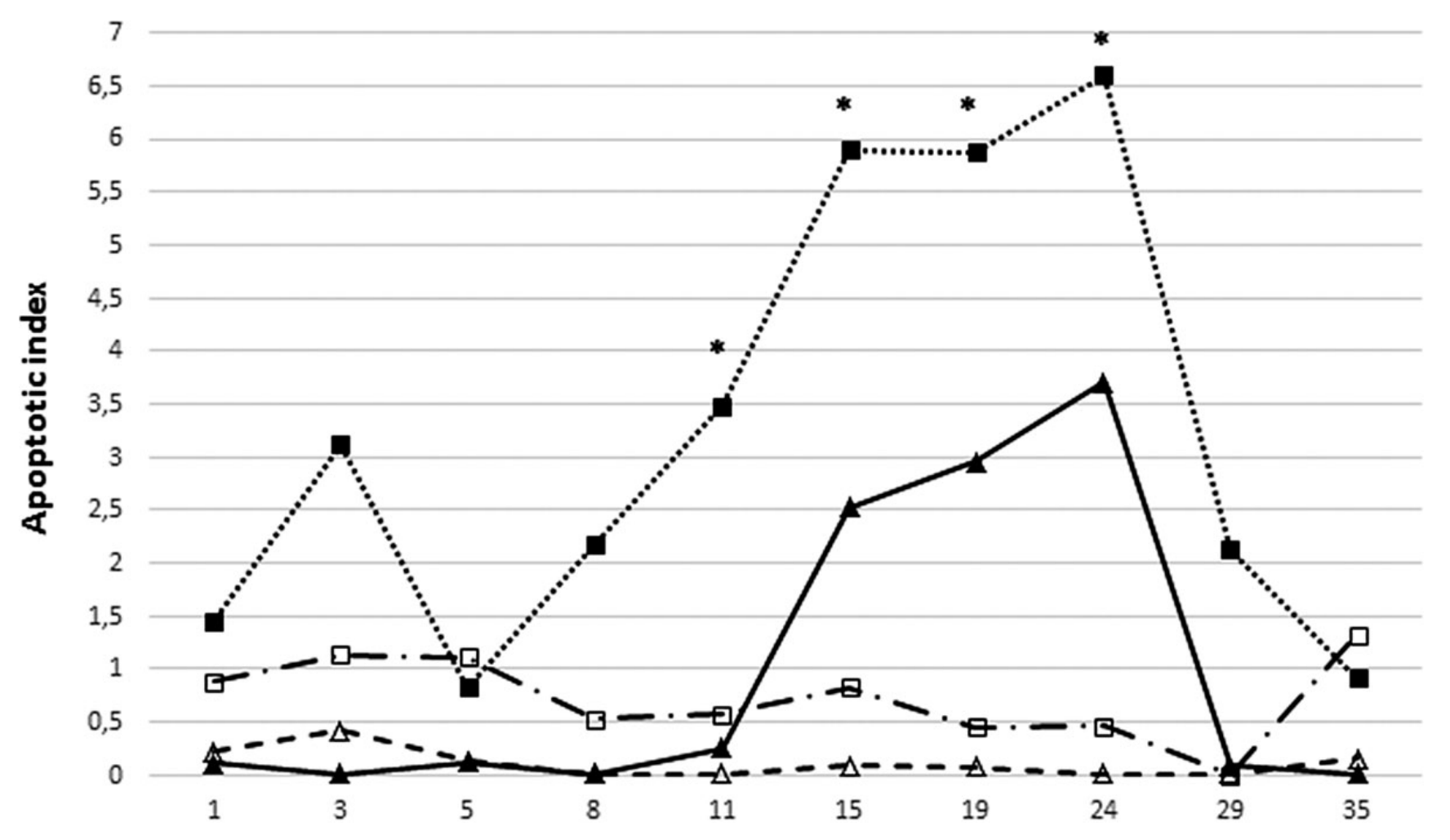

8

Days post infection

Figure 8. Temporal changes in the apoptotic index detected by the TUNEL and caspase-3 immunohistochemistry methods. Apoptotic events are higher at II, I 1 , 19, and 24 days postinfection in crypt cells of pigs experimentally inoculated with $L$. intracellularis. $* P<.05$. $\square=$ challenge group, results for caspase-3; $\square=$ control group, results for caspase-3; $\boldsymbol{\Lambda}=$ challenge group, results for TUNEL; $\Delta=$ control group, results for TUNEL.

24. PCR positivity by day 3 postinoculation was probably related to inoculum that was still passing through the intestinal tract. PCR positivity in fecal samples by day 35 postinoculation might be explained by $L$. intracellularis infection in the large intestine later in the course of the disease ( 29 days postinoculation) based on IHC findings and that it could be easily missed on day 35 using the same technique due to the length of the large intestine in comparison with the small samples collected for histology and IHC.

Only 1 challenged pig had a focal area of $L$. intracellularis infection in the duodenum, as demonstrated by IHC, whereas 12 had widespread staining in the large intestine. It is possible that conditions such as $\mathrm{pH}$ or lack of specific cellular receptors might be responsible for the lower susceptibility of the duodenum to L. intracellularis.

Detection of L. intracellularis-specific IgA in intestinal lavages was previously reported in experimentally infected pigs 22 days postinfection. ${ }^{7}$ In the present study, this procedure was performed in correlation with the disease progression. This immunoglobulin was detected between 15 and 29 days postinfection, and the highest titer was 1:16 at 24 days postinfection. This local humoral immune response appeared to be more short lived than the systemic humoral and cell-mediated immune responses, ${ }^{7}$ although the sensitivity of the IPMA for detecting IgA in intestine lavages is unknown. Moreover, due to the small sample size, no correlation analysis with PE lesions or L. intracellularis infection was performed. Further studies correlating the presence of local immune response and protection have yet to be conducted.

Decreased apoptosis has been suggested as one of the mechanisms by which L. intracellularis induces proliferation of infected enterocytes. ${ }^{21,24}$ However, based on TUNEL and caspase-3 IHC findings of the present study, it was demonstrated that $L$. intracellularis-infected enterocytes, in fact, have an increase in apoptotic events. It is interesting that the IHC score and histologic lesions increased in the periods in which AI was high. Therefore, we speculate that the proliferation rate overcomes the increase in apoptotic events. Oh et $\mathrm{al}^{23}$ suggest that pro apoptotic genes are upregulated in the first 48 hours post in vitro L. intracellularis infection in McCoy cells. It is important to remember that $L$. intracellularis infection in vitro does not result in cell proliferation, ${ }^{15}$ which makes it difficult to extrapolate these results to our in vivo study. On the other hand, Vannucci et $\mathrm{al}^{24}$ found reduced expression of genes involved in apoptosis at 21 days postinfection, the period in which gross and histologic lesions of $\mathrm{PE}$ are most extensive. ${ }^{9,24}$ The 

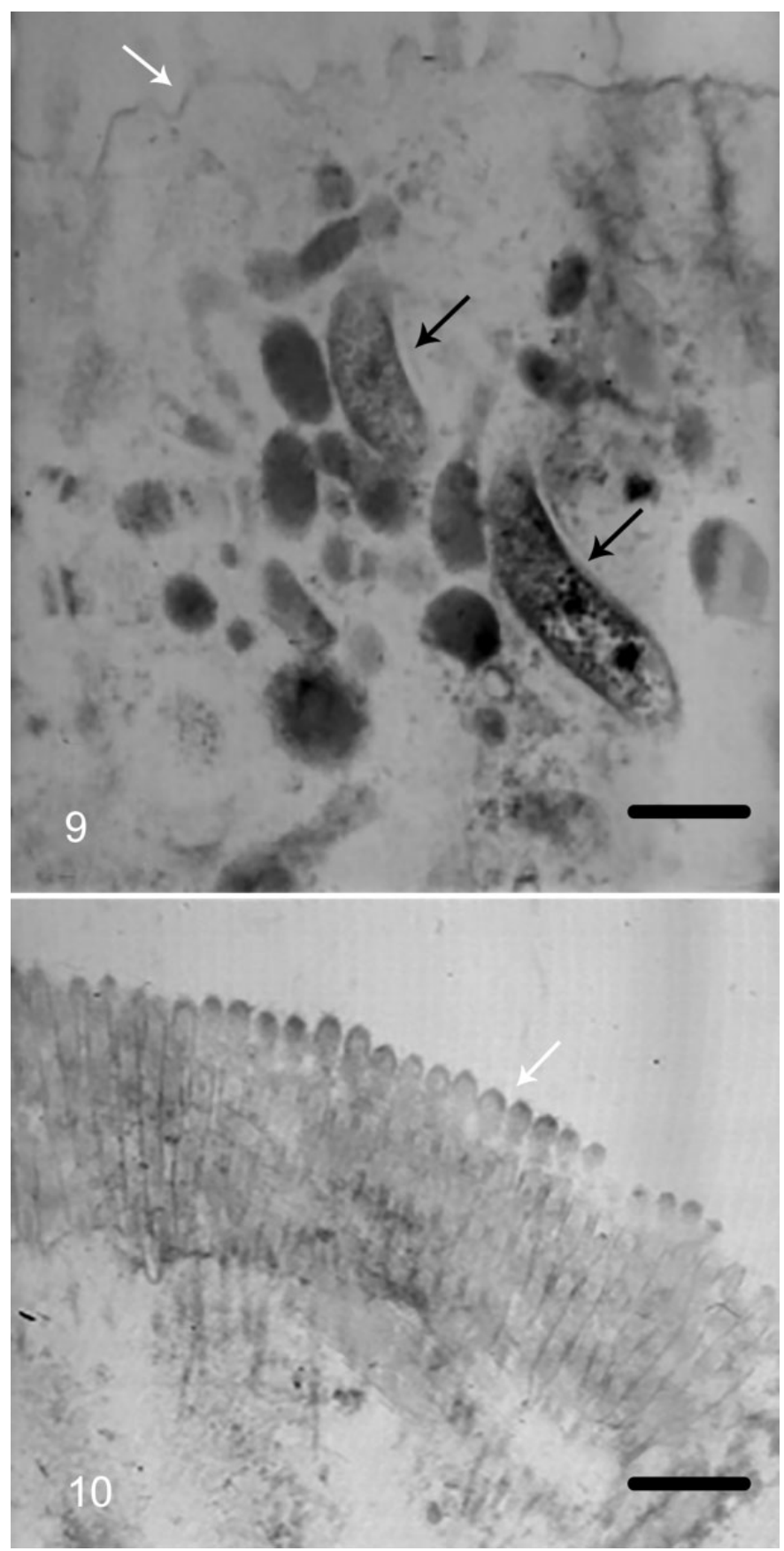

Figures 9, 10. Proliferative enteropathy, enterocytes, pig. Transmission electron microscopy. Figure 9. Day 15, oblique section of $2 \mathrm{~L}$. intracellularis organisms (black arrows) in the terminal web of immature enterocytes with poorly developed microvilli (white arrow). Bar $=0.285 \mu$. Figure 10. Day 24, note the length of fully developed microvilli (white arrow) in crypt enterocyte. Bar $=0.455 \mu$.

approach used by Vannucci et $\mathrm{al}^{24}$ proposed a decrease of apoptotic events at the molecular level, whereas our study demonstrated that there is an increase in apoptotic activity in the infected crypts. There is no clear explanation for this difference in findings. However, we hypothesize that although the transcription analysis has demonstrated stimuli to decrease

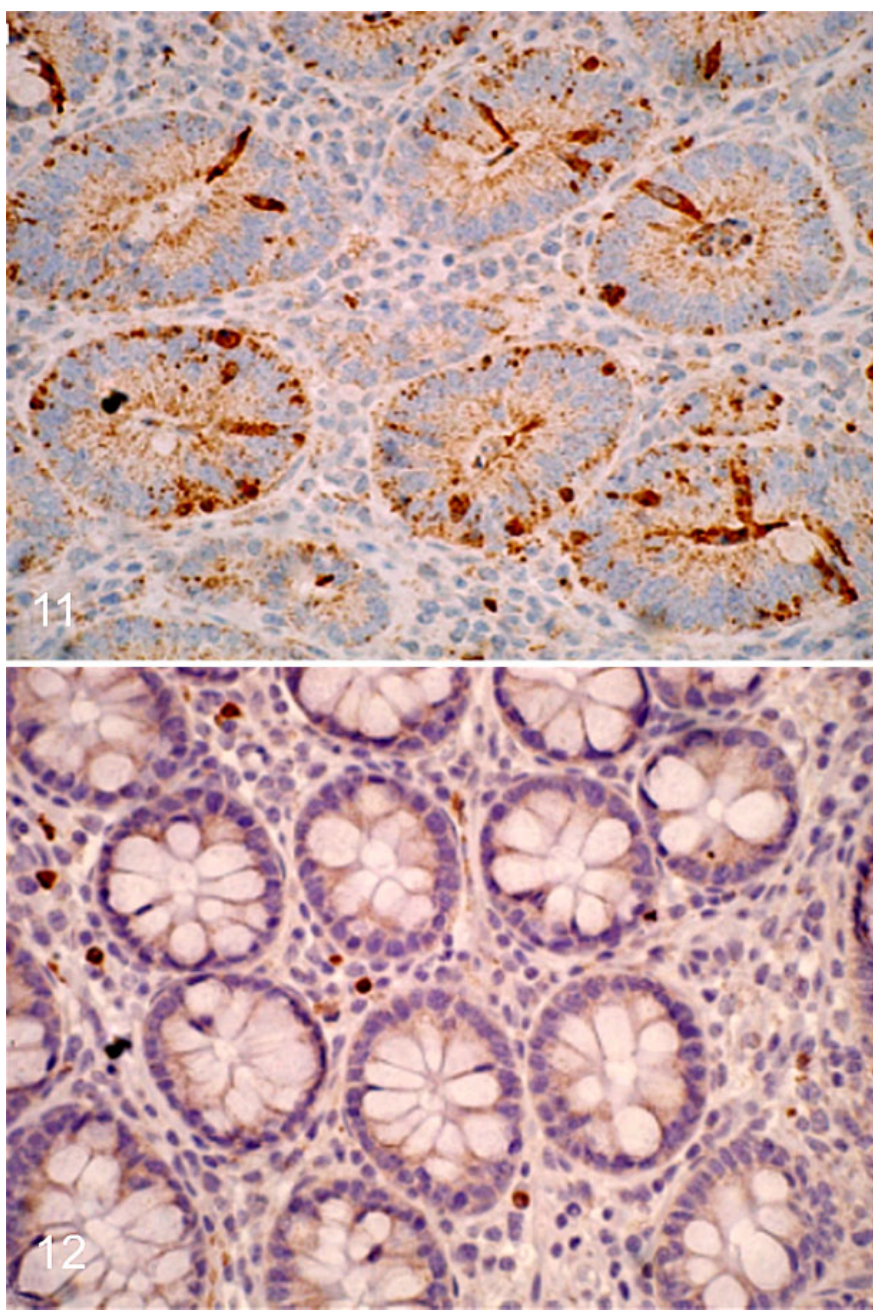

Figures I I, I 2. Proliferative enteropathy, ileum, pig, day 24. Immunohistochemistry for caspase-3. Figure II. Crypts with several immunolabeled immature cells with homogeneous diffuse cytosolic brown staining. Figure I2. Normal crypts with mature enterocytes and goblet cells and no immunolabeling.

Table 2. Fecal Shedding Detected by PCR and IgA Titers in Intestinal Lavage of Pigs at Different Time Points After Experimental Infection with Lawsonia intracellularis.

\begin{tabular}{|c|c|c|c|c|c|c|c|c|c|c|}
\hline & \multicolumn{10}{|c|}{ No. of Days After Inoculation } \\
\hline & 1 & 3 & 5 & 8 & II & 15 & 19 & 24 & 29 & 35 \\
\hline PCR & $-1-$ & $+1-$ & $-1-$ & $-1-$ & $+/+$ & $+1+$ & $+1+$ & $+1-$ & $-1-$ & $-1+$ \\
\hline $\lg A$ & NT & NT & NT & NT & $-1-$ & $-/ 1: 4$ & $\mathrm{I}: 4 / \mathrm{I}: 4$ & $\mathrm{I}: 16 / \mathrm{I}: 4$ & $-/ 1: 4$ & $-1-$ \\
\hline
\end{tabular}

Abbreviation: NT, not tested. The data are based on 2 pigs at each time point.

apoptosis, there is no direct correlation between the number of genes that are up- or downregulated and cell cycle change. In other words, there are other events posttranscription (translation, protein degradation, and protein interactions) that are important for regulating the cell cycle. Therefore, although Vannucci et $\mathrm{al}^{24}$ found an increase in expression of genes that are 
linked to decreased apoptosis, posttranscriptional events may result in programmed cell death. ${ }^{6,18}$

Similar to our findings about apoptosis events during $L$. intracellularis infection, Jones et $\mathrm{al}^{14}$ have found that Helicobacter pylori infection induces a reversible apoptosis; in other words, apoptotic events would return to basal levels after $H$. pylori elimination. A type III secretion system (T3SS) has been identified in L. intracellularis. ${ }^{1}$ Although there is not complete knowledge about its function and effector proteins in L. intracellularis, in other gram-negative bacterial species such as Vibrio alginolyticus, T3SS has been recognized as an important inductor of caspase-3 that, as a consequence, indirectly induces apoptosis. ${ }^{26}$ Citrobacter rodentium, a gram-negative bacterium that causes transmissible colonic hyperplasia in mice, also induces cell apoptosis, although not through the caspase- 3 pathway. ${ }^{5}$ Furthermore, this species also has a T3SS that delivers effector proteins to host cells, ${ }^{22}$ and these proteins have been described in $E$. coli as having a cytotoxic effect that could result in apoptosis. ${ }^{4}$ Further studies to identify the relationship between L. intracellularis T3SS effector proteins and apoptosis must be conducted. Furthermore, reduction of programmed cell death (apoptosis) is not the explanation for enterocyte hyperplasia, the hallmark lesion observed in L. intracellularis infection. It is possible that a host response as a result of intracellular $L$. intracellularis infection may play this role in the pathogenesis of PE.

\section{Acknowledgements}

We thank Dr Maria Isabel M. Coelho-Guedes, Dr Matti Kiupel, Keith Kinsley, and Debra Swanson for technical assistance.

\section{Declaration of Conflicting Interests}

The author(s) declared no potential conflicts of interest with respect to the research, authorship, and/or publication of this article.

\section{Funding}

The author(s) disclosed receipt of the following financial support for the research, authorship, and/or publication of this article: R.M.C. Guedes was supported by the Brazilian government sponsoring agency Conselho Nacional de Desenvolvimento Científico e Tecnológico $(\mathrm{CNPq})$ and Universidade Federal de Minas Gerais. Partial funding for the research was provided by grant 00-52100-9687 from the USDA-CSREES-IFAFS research initiative and Elanco Animal Health. R.M.C. Guedes has a research fellowship from CNPq.

\section{References}

1. Alberdi MP, Watson E, McAllister GE, et al. Expression by Lawsonia intracellularis of type III secretion system components during infection. Vet Microbiol. 2009;139(3-4):298-303.

2. Boutrup TS, Boesen HT, Boye M, et al. Early pathogenesis in porcine proliferative enteropathy caused by Lawsonia intracellularis. J Comp Pathol. 2010; 143(2-3):101-109.

3. Cooper DM, Gebhart CJ. Comparative aspects of proliferative enteritis. J Am Vet Med Assoc. 1998;212(9):1446-1451.

4. Crane JK, McNamara BP, Donnenberg MS. Role of EspF in host cell death induced by enteropathogenic Escherichia coli. Cell Microbiol. 2001;3(4):197-211.

5. Flynn AN, Buret AG. Tight junctional disruption and apoptosis in an in vitro model of Citrobacter rodentium infection. Microb Pathog. 2008;45(2):98-104.
6. Greenbaum D, Colangelo C, Williams K, et al. Comparing protein abundance and mRNA expression levels on a genomic scale. Genome Biol. 2003;4(9): 117.

7. Guedes RM, Gebhart CJ. Evidence of cell-mediated immune response and specific local mucosal immunoglobulin (Ig) A production against Lawsonia intracellularis in experimentally infected swine. Can J Vet Res. 2010;74(2): 97-101.

8. Guedes RM, Gebhart CJ. Comparison of intestinal mucosa homogenate and pure culture of the homologous Lawsonia intracellularis isolate in reproducing proliferative enteropathy in swine. Vet Microbiol. 2003;93(2):159-166.

9. Guedes RM, Gebhart CJ. Onset and duration of fecal shedding, cell-mediated and humoral immune responses in pigs after challenge with a pathogenic isolate or attenuated vaccine strain of Lawsonia intracellularis. Vet Microbiol. 2003; 91(2-3):135-145.

10. Guedes RM, Gebhart CJ. Preparation and characterization of polyclonal and monoclonal antibodies against Lawsonia intracellularis. $J$ Vet Diagn Invest. $2003 ; 15(5): 438-446$

11. Guedes RM, Gebhart CJ, Deen J, et al. Validation of an immunoperoxidase monolayer assay as a serologic test for porcine proliferative enteropathy. $J$ Vet Diagn Invest. 2002;14(6):528-530.

12. Guedes RM, Gebhart CJ, Winkelman NL, et al. Comparison of different methods for diagnosis of porcine proliferative enteropathy. Can J Vet Res. 2002; 66(2):99-107.

13. Jones GF, Ward GE, Murtaugh MP, et al. Enhanced detection of intracellular organism of swine proliferative enteritis, ileal symbiont intracellularis, in feces by polymerase chain reaction. J Clin Microbiol. 1993;31(10):2611-2615.

14. Jones NL, Shannon PT, Cutz E, et al. Increase in proliferation and apoptosis of gastric epithelial cells early in the natural history of Helicobacter pylori infection. Am J Pathol. 1997;151(6):1695-1703.

15. Lawson GH, Gebhart CJ. Proliferative enteropathy: review. J Comp Pathol. 2000;122(2-3):77-100.

16. Lawson GH, McOrist S, Jasni S, et al. Intracellular bacteria of porcine proliferative enteropathy: cultivation and maintenance in vitro. J Clin Microbiol. 1993;31(5):1136-1142.

17. MacIntyre N, Smith DG, Shaw DJ, et al. Immunopathogenesis of experimentally induced proliferative enteropathy in pigs. Vet Pathol. 2003;40(4): 421-432.

18. Maier T, Guell M, Serrano L. Correlation of mRNA and protein in complex biological samples. FEBS Lett. 2009;583(24):3966-3973.

19. McOrist S, Gebhart CJ. Porcine proliferative enteropathies. In: D’Allaire BE, Mengeling S, Taylor DJ, eds. Diseases of Swine. 8th ed. Ames, IA: Iowa State University Press; 1999:521-534.

20. McOrist S, Jasni S, Mackie RA, et al. Entry of the bacterium ileal symbiont intracellularis into cultured enterocytes and its subsequent release. Res Vet Sci. 1995;59(3):255-260.

21. McOrist S, Roberts L, Jasni S, et al. Developed and resolving lesions in porcine proliferative enteropathy: possible pathogenetic mechanisms. J Comp Pathol. 1996;115(1):35-45.

22. Mundy R, Petrovska L, Smollett K, et al. Identification of a novel Citrobacter rodentium type III secreted protein, EspI, and roles of this and other secreted proteins in infection. Infect Immun. 2004;72(4):2288-2302.

23. Oh YS, Lee JB, McOrist S. Microarray analysis of differential expression of cell cycle and cell differentiation genes in cells infected with Lawsonia intracellularis. Vet J. 2010;184(3):340-345.

24. Vannucci FA, Foster DN, Gebhart CJ. Laser microdissection coupled with RNA-seq analysis of porcine enterocytes infected with an obligate intracellular pathogen (Lawsonia intracellularis). BMC Genomics. 2013;14:421. doi:10.1186/1471-2164-14-421.

25. Vannucci FA, Gebhart CJ. Recent advances in understanding the pathogenesis of Lawsonia intracellularis infections. Vet Pathol. 2014;51(2):465-477.

26. Zhao Z, Chen C, Hu CQ, et al. The type III secretion system of Vibrio alginolyticus induces rapid apoptosis, cell rounding and osmotic lysis of fish cells. Microbiology. 2010;156(Pt 9):2864-2872. 